\title{
NUEVOS CULTIVARES DE MELÓN (Cucumis melo L.) PARA INVERNADERO EN PUERTO LA BOCA, MANABÍ.
}

\author{
AUTORES: Julio Gabriel Ortega ${ }^{1}$ \\ Joel Banchón Toro² \\ Fernando Ayón Villao ${ }^{3}$ \\ Máximo Vera Tumbaco ${ }^{4}$ \\ Washington Narváez Campana ${ }^{5}$
}

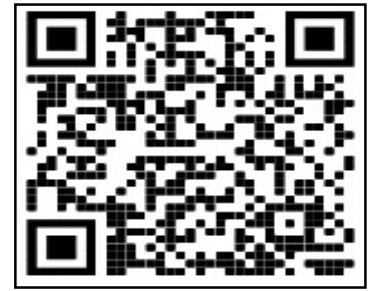

\section{DIRECCIÓN PARA CORRESPONDENCIA: julio.gabriel@unesum.edu.ec}

Fecha de recepción: 10/04/2020

Fecha de aceptación: 26/11/2020

\section{RESUMEN}

La presente investigación de nuevos cultivares de melón (Cucumis melo L.) para cultivo invernadero para Puerto La Boca, Manabí, tuvo como objetivos: i) Evaluar y seleccionar cultivares de melón (Cucumis melo L.) en condiciones de invernadero, ii) realizar un análisis parcial de costo/beneficio de tratamientos objetos de estudio. Se implementó en invernadero una parcela en diseño experimental de parcelas dividas en franja con cuatro repeticiones, donde los tratamientos fueron los cultivares [A1: karametza, A2: kazta, A3: kapaz, A4: kazik y A5: primo (testigo)] y los subtratamientos los tipos de manejo (B1: Tutorado y B2: Rastero). Las variables de respuesta evaluadas fueron: Peso de frutos (g), número de frutos comerciales por planta, volumen total de frutos (cm3) y una estimación económica de tratamientos. Los resultados permitieron concluir que el melón con mejor promedio de número de frutos fue donde se utilizó sistema rastrero con 1,95 en promedio, y el mayor peso de frutos (g) correspondió al sistema de tutorado con 1,06 en promedio. Los mejores cultivares fueron Kapaz y kazik, y el mejor sistema fue el tutorado. La estimación económica mostró que los cultivares fueron rentables cuando se vendieron a supermercado con una relación B/C entre \$2,17 y \$2,03 respectivamente.

Palabras claves: Producción, tolerancia, resistencia, volumen, peso.

1 Profesor investigador, Carrera Agropecuaria, Facultad de Ciencias Naturales y de la Agricultura, Universidad Estatal del Sur de Manabí, Km 11\%2 vía Jipijapa-Noboa - Campus los Ángeles. Correo- e: julio.gabriel@unesum.edu.ec. https://orcid.org/0000-0001-9776-9235

2 Funcionario Gobierno Autónomo Descentralizado de Paján, Manabí. Correo-e: JOEL_14TORETTO@hotmail.com. https://orcid.org/00000003-0578-3860

3 Profesor investigador, Carrera Agropecuaria, Facultad de Ciencias Naturales y de la Agricultura, Universidad Estatal del Sur de Manabí, Km 11⁄2 vía Jipijapa-Noboa - Campus los Ángeles. Correo- e: fernando.ayon @unesum.edu.ec. https://orcid.org/0000-0003-4772-9344

${ }^{4}$ Profesor investigador, Carrera Agropecuaria, Facultad de Ciencias Naturales y de la Agricultura, Universidad Estatal del Sur de Manabí, Km 11⁄2 vía Jipijapa-Noboa - Campus los Ángeles. Correo- e: maximo.vera@unesum.edu.ec. https://orcid.org/0000-0003-2320-712X

5 Profesor investigador, Carrera Agropecuaria, Facultad de Ciencias Naturales y de la Agricultura, Universidad Estatal del Sur de Manabí, Km 1² vía Jipijapa-Noboa - Campus los Ángeles. Correo- e: washington.narvaez@unesum.edu.ec. https://orcid.org/0000-0002-6674-2088 


\title{
NEW MELON CULTIVARS (Cucumis melo L.) FOR GREENHOUSE IN PUERTO LA BOCA, MANABÍ.
}

\begin{abstract}
The present investigation of new cultivars of melon (Cucumis melo L.) for greenhouse cultivation for Puerto La Boca, Manabí, had as objectives: i) Evaluate and select cultivars of melon (Cucumis melo L.) in greenhouse conditions, ii) perform a partial cost/benefit analysis of the treatments under study. A plot in an experimental design of plots divided into strips with four replications was implemented in a greenhouse, where the treatments were the cultivars [A1: karametza, A2: kazta, A3: kapaz, A4: kazik and A5: primo (control)] and the subtreatments the types of management (B1: Tutored and B2: Creeping). The response variables evaluated were: Weight of fruits (g), number of commercial fruits per plant, total volume of fruits $(\mathrm{cm} 3)$ and an economic estimate of treatments. The results allowed to conclude that the melon with the best average number of fruits was where the creeping system was used with 1,95 on average, and the highest weight of fruits (g) corresponded to the tutored system with 1,06 on average. The best cultivars were Kapaz and kazik, and the best system was the tutored one. The economic estimation showed that the cultivars were profitable when sold to the supermarket with a B / C ratio between $\$ 2,17$ and $\$ 2,03$ respectively.
\end{abstract}

Keywords: Production, tolerance, resistance, volume, weight.

\section{INTRODUCCIÓN}

El Ecuador dispone de condiciones ambientales favorables para el cultivo de una infinidad de especies vegetales que pueden ser consideradas como hortalizas, tanto en la Sierra como en la Costa. Según estadísticas del proyecto para la "Reorientación del sector agropecuario", del Ministerio de Agricultura y Ganadería, en el Ecuador se dedican en la actualidad alrededor de 40.000 hectáreas al cultivo de hortalizas, siendo las de mayor importancia por área sembrada: cebolla colorada 7.920 ha, tomate riñón 7.560 ha, cebolla blanca 4.230 ha, sandía 3.860 ha, melón 3.430 ha y zanahoria amarilla 2.800 ha (Ramírez, 2014).

El melón es una planta cuyos frutos contienen propiedades nutricionales y son consumidos en fresco, así como en la elaboración de dulces, conservas y congelados. Es un cultivo importante para la exportación, genera divisas al país y mayores ingresos económicos a los productores (Carrillo et al., 2010). En Ecuador la explotación de melón (Cucumis melo L.) en las últimas décadas ha tenido un auge notable, ocupando el segundo lugar por superficie sembrada entre las cucurbitáceas. Generalmente en el litoral ecuatoriano se cultiva desde diciembre hasta marzo (época invernal), con una superficie de 924 hectáreas y una producción de 7.549 toneladas, convirtiéndose en un producto de interés comercial en el país; se exporta a los países europeos el $1,4 \%$ del total de la producción.

En el Ecuador existen regiones con gran potencial para el desarrollo del cultivo, sobre todo áreas de alta luminosidad y temperaturas, como el valle del río Portoviejo en la provincia de Manabí y el cantón Santa Elena en la provincia de Santa Elena (Orrala et al., 2013) En Manabí el cultivo del melón está muy difundido con el uso de cultivares tradicionales, aunque en los últimos años ciertos horticultores vienen empleando semillas mejoradas incrementando con esta práctica el 2 
rendimiento y calidad de los frutos. Entre los cultivares híbridos más comunes utilizados se cita: Edisto 47, Pacstar, Primo, Excelsior, Honey Dew y el criollo (Carrillo et al., 2010).

Según datos de información acuícola, agrícola, ganadera y pesquera del Ecuador, en Manabí se sembraron alrededor de 896 has en el año 2017, el melón sembrado en su mayoría es el Cantaloupe porque es el más consumido en el país (Gómez, 2017). En el cantón Jipijapa una de las productoras de melón, está ubicada en la Parroquia Puerto Cayo, específicamente en las localidades que comprenden el valle de Canta Gallo y Puerto La Boca. En estos sitios se cultiva el melón a campo abierto y desde hace unos tres años atrás se está incentivando la siembra en invernadero y el uso de semillas mejoradas, lo que está incrementando la producción por un manejo eficiente y adecuado.

En Puerto la Boca el Ministerio de Agricultura y Ganadería incentivo a los agricultores para que mejoren su producción de hortalizas con la implementación de invernaderos, aquí se construyeron un total de 54 invernaderos de los cuales actualmente están en producción 34. La inversión por invernadero fue de USD 4.000 y lo cual tuvieron los agricultores que ubicar una contraparte de USD 560 dólares por cada invernadero implementado, con un total 16.416 dólares por productor, además con la estimación de los valores de las fincas aproximadamente en USD 3.000 la hectárea (Gabriel et al. 2020a).

\section{MATERIALES Y MÉTODOS}

\section{Ubicación}

La investigación se desarrolló en un invernadero de $500 \mathrm{~m}^{2}$ ubicado en el Recinto Puerto la Boca de la Parroquia Puerto Cayo, Cantón Jipijapa, que está ubicado a 45 minutos de Jipijapa $\left(1^{\circ} 18 ' 20^{\prime \prime S}, 80^{\circ} 45^{\prime} 42^{\prime \prime} \mathrm{O}\right.$ y altura de $\left.53 \mathrm{msnm}\right)$; su clima posee una temperatura de $24,8{ }^{\circ} \mathrm{C}$; la precipitación promedio anual es de $298 \mathrm{~mm}$, concentrándose la mayor cantidad de lluvia en el mes de febrero, mientras que el mes más seco es agosto (Gabriel et al., 2020b).

\section{Tratamientos y diseño experimental}

Los tratamientos fueron constituidos por los cultivares (A1: Karmetza, A2: Kazta, A3: Kapaz, A4: Kazik; A5: Primo y los subtratamientos por el tipo de manejo (B1: Tutorado, B2: Rastrero). Los cultivares fueron provistos por la empresa holandesa Enza Zaden (2018).

El experimento fue implementado en un invernadero de $500 \mathrm{~m}^{2}$ en un diseño de parcelas dividida en franja con cuatro repeticiones (Gabriel et al. 2017). La distancia entre hileras fue de 1,5 m, la distancia entre plantas fue de $0,50 \mathrm{~m}$ y la distancia entre unidades experimentales de 2,5 $\mathrm{m}$. Cada unidad experimental estuvo constituida de 3 hileras y 30 plantas. Se eligieron en cada unidad experimental 10 plantas al azar de la parte central para las evaluaciones y evitar el efecto de bordura. El experimento fue de $450 \mathrm{~m}^{2}$

\section{Análisis estadísticos}

Sobre la base del modelos definido y previo análisis de normalidad y homogeneidad de varianza, se realizó análisis de varianza, para probar hipótesis de los efectos fijos, así como la comparación de medias de los tratamientos mediante la prueba de tukey al $\operatorname{Pr}<0,05$ de probabilidad. El análisis 
de varianza también sirvió para estimar los componentes de varianza para los efectos aleatorios. Los análisis indicados se realizaron utilizando el Proc GLM del SAS University (SAS 2019),

\section{Análisis de correlación}

Se procedió a realizar el análisis de correlación entre las variables de respuesta correspondientes utilizando el coeficiente de correlación de Pearson (Gabriel et al. 2017).

\section{Variables de respuesta}

Peso de frutos (g). Se pesaron los frutos en una balanza calibrada en kilogramos de cada planta tomada dentro de la parcela útil.

Número de frutos comerciales por planta $\left(\mathbf{N}^{\circ}\right)$. Se contabilizaron el número de frutos comerciales por planta dentro de la parcela útil para determinar el rendimiento de frutos sanos por cada pase de cosecha.

Largo de frutos $(\mathbf{c m})$. El largo de frutos se midió con la ayuda de una cinta métrica, se tomó uno a uno los frutos para sacar este dato.

Ancho de frutos (cm). El ancho de frutos se tomó considerando en la parte intermedia del fruto con la ayuda de una cinta métrica.

Alto de frutos (cm). El alto del fruto se tomó con la ayuda de una cinta métrica desde la base hasta la parte más alta por tratamiento.

Volumen total de frutos $\left(\mathbf{c m}^{3}\right)$. Para determinar el volumen de cada fruto se utilizó la formula recomendada por Martel Moreno (1999):

\section{Beneficio/Costo.}

Para la relación B/C se determinó el beneficio de la tecnología, en su interpretación se consideró si el resultado fue mayor a 1 (Boardman 2006), este fue aceptable o rentable, si el resultado fue igual a 1 , lo que indicó fue que no tuvo beneficio de lucro ni pérdida y si el resultado fue menor a 1 implicó que no fue rentable, por lo cual el proyecto sería rechazado.

\section{Manejo de la investigación}

La pre-germinación de la semilla, se hizo en tarrinas plásticas transparentes con papel tolla humedecida con agua destilada, sobre las cuales se vertió 100 semillas por cultivar. A los cinco días después de la germinación (ddg), las plántulas fueron transplantadas a bandejas de almácigo preparadas (tierra del lugar, tierra de guaba, biocompost en una proporción 1:1:2 y 200 g de micorriza). El transplante fue realizado con pinzas quirúrgicas, teniendo cuidado de no lastimar las plántulas. Las bandejas se dejaron bajo invernadero durante 20 días. El riego se hizo dos veces por día.

El trasplante definitivo se realizó a los 20 ddg. El control de malezas fue manual. El riego por goteo fue realizado uno en la mañana $(30 \mathrm{~min})$ y otro en la tarde (30 min). Se realizó la fertilización foliar a los 35 días después del trasplante (ddt) con $4 \mathrm{~kg}$ de nitrato de potasio cristalizado + magnesio en una dosis de $2 \mathrm{~kg}$ de nitrato de potasio y $2 \mathrm{~kg}$ de sulfato de magnesio. Asimismo, se fertilizó con solufol desarrollo (100 g/15 l), durante cuatro ocasiones cada ocho 
días. A los 50 ddt se aplicó en forma diluida dos libras de $\mathrm{KNO}_{3}$, dos libras de $\mathrm{CO}\left(\mathrm{NH}_{2}\right)_{2}$ (urea) y dos libras $\mathrm{MgSO}_{4}$.

Para el control de insectos se aplico Acetamiprid a los 10ddt (40 g/15 l). Asimismo se aplicó Bacillus thurigiensis a los 20 y 50 ddt $(80 \mathrm{~g} / 15 \mathrm{l})$. Para el control del mildiu velloso (Pseudoperonosphora cubensis), se aplicó Metalaxy + Mancozeb (70 g/15 l) a los 8 y 30 ddt. A los 60 ddt se realizó la cosecha por planta en la parcela útil.

\section{RESULTADOS Y DISCUSIÓN}

\section{Análisis de normalidad}

El análisis de normalidad mostró que $\mathrm{A}=0$ (sesgo) el $\mathrm{K}<1$ (curtosis) y el C.V. = 33,75\%, estuvo dentro de los rangos permitidos para este tipo de investigación. La prueba Kolmogorov-Smirnov mostró diferencias significativas al $\operatorname{Pr}<0,05$ de probabilidad, lo que denotó un ajuste normal de los datos (Tabla 1).

Tabla 1. Análisis de normalidad de los tratamientos.

\begin{tabular}{lc}
\hline & Momentos \\
\hline Desviación & 0,50 \\
Varianza & 0,26 \\
Sesgo & 0 \\
Curtosis & $-2,10$ \\
Coeficiente de variación (C.V.) & 33,75 \\
Kolmogorov-Smirnov & $0,34^{* *}$ \\
\hline
\end{tabular}

\section{Análisis de la homogeneidad de varianza}

El análisis de homogeneidad de varianzas mediante la prueba de chi-cuadrada mostró que las medias de varianza fueron significativas al $\operatorname{Pr}<0,01$ de probabilidad, indicando esto que las medias fueron homogéneas, por lo que se vio por conveniente seguir con el análisis de varianza.

\section{Análisis de varianza}

El Análisis de varianza para el promedio de número de frutos (PNF): Peso de frutos (g) (PF) y volumen de frutos $\left(\mathrm{cm}^{3}\right)(\mathrm{VF})$, indica que los C.V. están dentro de los rangos permitidos para este tipo de investigación (C.V. de 14,24 a 25,91\%). El coeficiente de determinación ( $\mathrm{R}^{2}$ ) fue de 0,72; 0,61 y 0,52 respectivamente, lo que indica que los datos explican el modelo en $62 \%$ y el $38 \%$ restante se deben a factores ambientales (Tabla 2). 
Tabla 2. Análisis de varianza para promedio de número de frutos, peso de frutos (g) y volumen de frutos $\left(\mathrm{cm}^{3}\right)$.

\begin{tabular}{lcllc}
\hline \multicolumn{1}{c}{$\mathbf{F V}$} & gl & PNF & PF & VF \\
\hline Total & 39 & & & \\
Rep & 3 & $0,02 \mathrm{~ns}$ & $0,34 * *$ & $176717854,9 *$ \\
Trat & 1 & $3,05 * *$ & $0,32 *$ & $64887759,8 \mathrm{~ns}$ \\
Rep*Trat & 3 & $0,01 \mathrm{~ns}$ & $0,14 \mathrm{~ns}$ & $74805652,4 \mathrm{~ns}$ \\
Subtrat & 4 & $0,04 \mathrm{~ns}$ & $0,09 \mathrm{~ns}$ & $35358708,9 \mathrm{~ns}$ \\
Trat*Subtrat & 4 & $0,03 \mathrm{~ns}$ & $0,02 \mathrm{~ns}$ & $13236370,9 \mathrm{~ns}$ \\
Error & 24 & 0,06 & 0,06 & 39677361,0 \\
C.V. (\%) & & 14,2 & 25,05 & 25,91 \\
$\mathrm{R}^{2}$ & & 0,72 & 0,61 & 0,52 \\
\hline
\end{tabular}

*: Significativo al $\mathrm{P}<0.05$ de probabilidad, **: Altamente significativo al 0,01 de probabilidad, ns: No significativo, PNF: Promedio de Número de Frutos, PF: Peso de frutos, VF: Volumen de frutos.

El Análisis de varianza (ANVA) para PNF muestra que los cuadrados medios fueron altamente significativos a la probabilidad de $\operatorname{Pr}<0.01$ y para el PF el cuadrado medio fue significativo a la probabilidad de $\operatorname{Pr}<0,05$ (Tabla 4). Esto está indicando que al menos un tratamiento es diferente. Las otras fuentes de variación no fueron significativas (Tabla 2).

\section{Comparación de medias}

La comparación de medias para PNF, fue realizada mediante la prueba múltiple de tukey. El análisis mostró diferencias altamente significativas a la probabilidad de $\mathrm{P}<0,01$ (Tabla 3 ) donde se observó que el mejor tratamiento fue cuando se utilizó el sistema rastrero respecto al sistema tutorado. El PF mostró diferencias significativas al $\mathrm{P}<0,05$ de probabilidad, sobresaliendo el tratamiento donde se usó el tutorado en comparación con el sistema rastrero (Tabla 2). En la comparación de medias para volumen no se observaron diferencias significativas entre ambos sistemas de manejo.

Tabla 5. Comparación de medias para promedio de número de frutos, PF: peso de frutos (g) y volumen de frutos $(\mathrm{cm} 3)$ para tratamientos.

\begin{tabular}{lccc}
\hline Tratamiento & PNF & PF & V \\
\hline Rastrero & $1,94 \mathrm{a}$ & $0,89 \mathrm{~b}$ & $25511 \mathrm{a}$ \\
Tutorado & $1,38 \mathrm{~b}$ & $1,06 \mathrm{a}$ & $23101 \mathrm{a}$ \\
DSH & 0,15 & 0,16 & 4111.1
\end{tabular}

Medias con la misma letra no son significativamente diferentes al $\operatorname{Pr}<0.05$ de probabilidad, PNF: Promedio de Número de Frutos, PF: Peso de frutos, VF: Volumen de frutos

\section{Comparación de medias de los subtratamientos}


El análisis de medias de los subtratamientos (Tabla 4) mostró que no hubo diferencias significativas para PNF, PF y V. esto está indicando que los cultivares híbridos no fueron diferentes significativamente al $\mathrm{Pr}<0.05$ de probabilidad, respecto al testigo Primo y tampoco entre sí. Esto está indicando que todos los cultivares tuvieron igual comportamiento.

Tabla 4. Comparación de medias promedio de número de frutos, peso de frutos (g) y volumen de frutos $\left(\mathrm{cm}^{3}\right)$ para subtratamientos.

\begin{tabular}{lccc}
\hline Subtratamiento & PNF & PF & V \\
\hline Kapaz & $1,74 \mathrm{a}$ & $1,08 \mathrm{a}$ & $24806 \mathrm{a}$ \\
Karametza & $1,71 \mathrm{a}$ & $0,83 \mathrm{a}$ & $21081 \mathrm{a}$ \\
Kazik & $1,69 \mathrm{a}$ & $1,06 \mathrm{a}$ & $26875 \mathrm{a}$ \\
Primo & $1,65 \mathrm{a}$ & $0,96 \mathrm{a}$ & $23620 \mathrm{a}$ \\
Kazta & $1,51 \mathrm{a}$ & $0,92 \mathrm{a}$ & $24901 \mathrm{a}$ \\
DSH & 0,35 & 0,36 & 9307.9 \\
\hline
\end{tabular}

Medias con la misma letra no son significativamente diferentes, PNF: Promedio de Número de Frutos, PF: Peso de frutos (g), VF: Volumen de frutos $\left(\mathrm{cm}^{3}\right)$.

\section{Análisis de correlación}

El análisis de correlación mediante el coeficiente de Pearson (Tabla 5), mostró una alta correlación positiva y significativa al $\operatorname{Pr}<0,05$ entre NP con NF $(0,89)$ y una alta correlación positiva y altamente significativa al $\operatorname{Pr}<0,01$ del NF con PNF (0,72). Esto está indicando que a mayor NP abra mayor NF y que el PNF será mayor a medida que haya mayor NF.

Tabla 5. Análisis de correlación mediante el coeficiente de Pearson

\begin{tabular}{lllll}
\hline & NP & NF & PNF & V \\
\hline NP & 1,00 & $0,89 * *$ & $0,35^{*}$ & $0,05 \mathrm{~ns}$ \\
NF & & 1,00 & $0,72^{* *}$ & $-0,04 \mathrm{~ns}$ \\
PNF & & 1,00 & $-0,14^{*}$ \\
V & & & 1,00 \\
\hline$* *:$ Altamente significativo al Pr<0.01 de probabilidad, *: Significativo al Pr<0,05 de \\
probabilidad, NP: Número de plantas, NF: Número de frutos por planta, PNF: Promedio de \\
Número de Frutos, VF: Volumen de frutos $\left(\mathrm{cm}^{3}\right)$
\end{tabular}

\section{Análisis económico}

La Figura 1, se muestra la comparación de medias para PNF y PF, donde se observó una mayor producción para el cultivar Kapaz. 


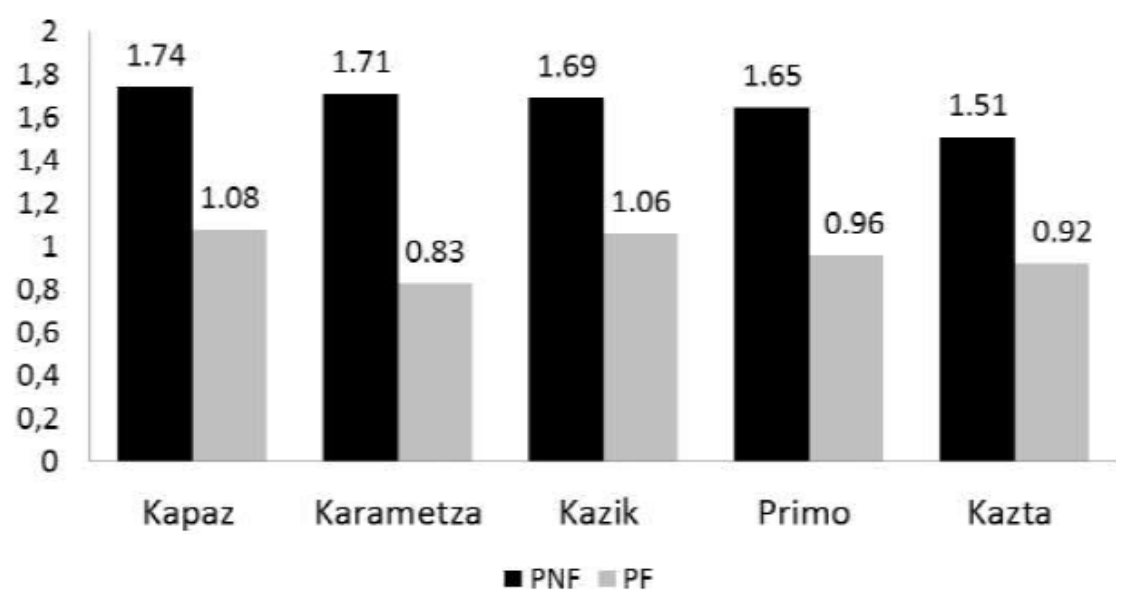

Figura 1. Comparación de medias para PNF: promedio de número de frutos, PF y peso de frutos (g) para tratamientos.

En la figura 2, se presenta los promedios para PNF: promedio de número de frutos y PF: peso de frutos (g) para tratamientos de melón rastrero y tutorado, donde se observó mayor PNF en el rastero, pero mejor PF en el tutorado.

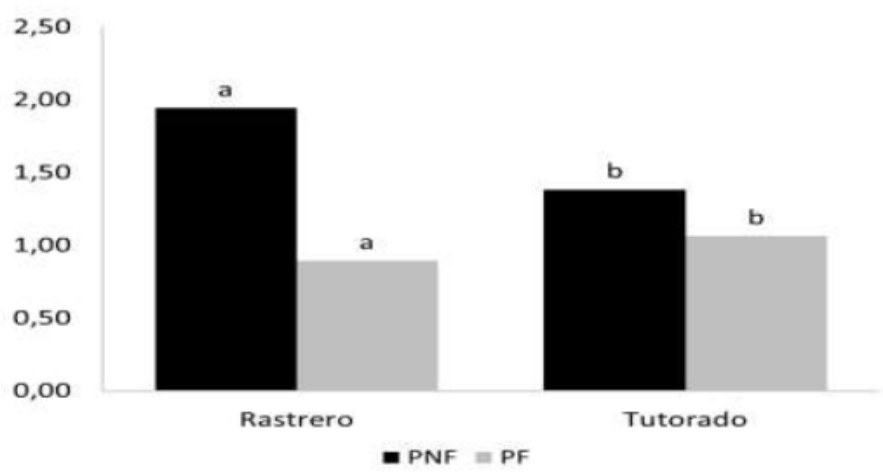

Figura 2. Comparación de medias para PNF: promedio de número de frutos y PF: peso de frutos (g) para tratamientos rastreos y con tutores.

En la tabla 6, se presenta el índice de rentabilidad económica de los ensayos, donde se puede notar que los tratamientos estudiados tienen una Relación B/C entre \$2,17 y \$1,35, que están por encima de $\$ 1,00$. 
Tabla 6. Índice de rentabilidad económica de los ensayos.

\begin{tabular}{|c|c|c|c|c|c|c|c|c|c|}
\hline 胥 & 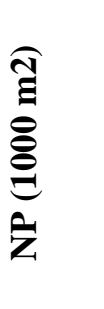 & 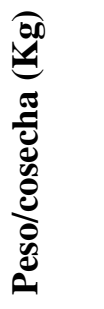 & 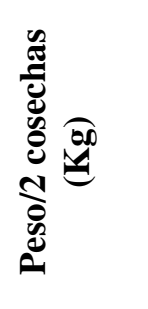 &  & 胥 & 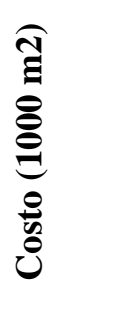 & 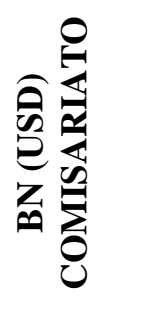 & 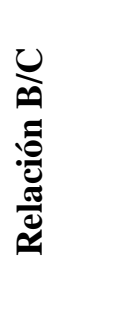 & 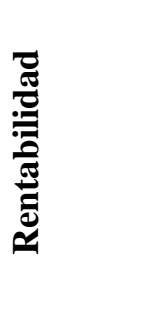 \\
\hline Kapaz & 1254 & 1,88 & 2356,52 & 0,5 & 1178,26 & 371,15 & 807,11 & 2,17 & Rentable \\
\hline Kazik & 1254 & 1,79 & 2246,42 & 0,5 & 1123,21 & 371,15 & 752,06 & 2,03 & Rentable \\
\hline Primo & 1254 & 1,58 & 1986,34 & 0,5 & 993,70 & 371,15 & 622,02 & 1,68 & Rentable \\
\hline Karametza & 1254 & 1,42 & 1779,80 & 0,5 & 889,90 & 371,15 & 518,75 & 1,40 & Rentable \\
\hline Kazta & 1254 & 1,39 & 1742,06 & 0,5 & 871,03 & 371,15 & 499,88 & 1,35 & Rentable \\
\hline
\end{tabular}

$\mathrm{B} / \mathrm{C}>1,00$

La figura 3, presenta la rentabilidad del cultivo de melón, donde se puede observar que existe una buena rentabilidad cuando se vende a los comisariatos y no es rentable con precios del mercado común.

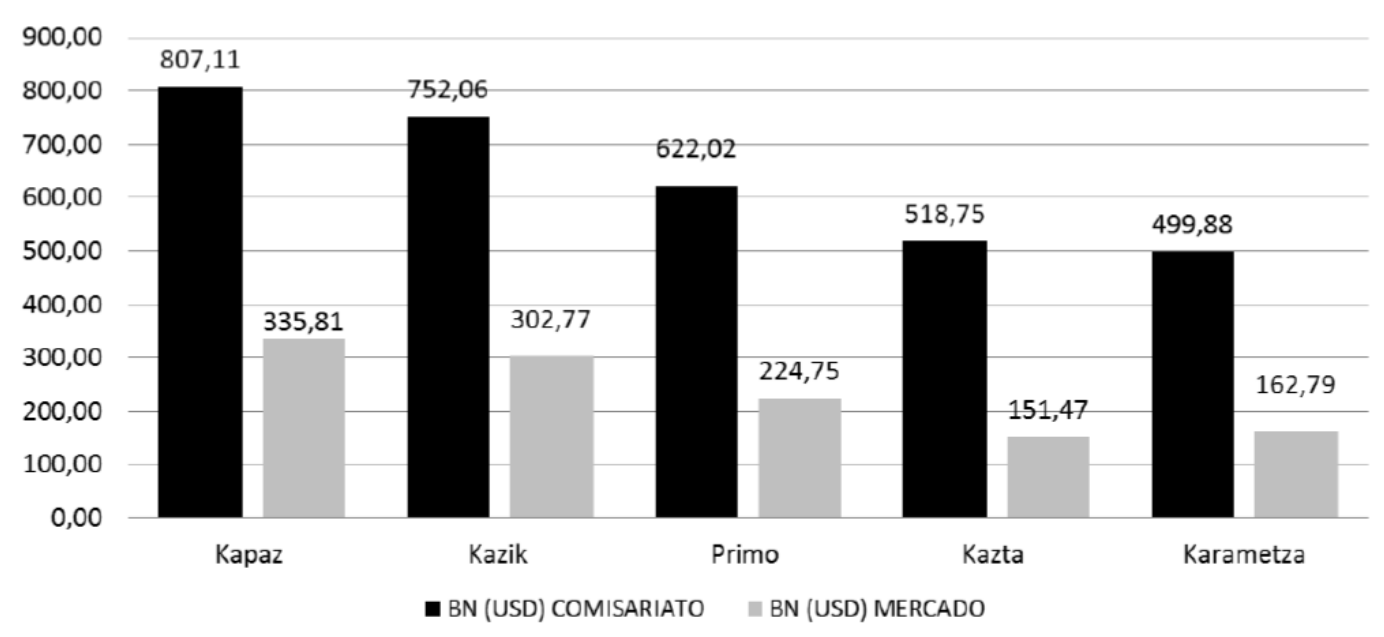

Figura 3. Análisis de rentabilidad con venta de melón a comisariato y al mercado común.

\section{DISCUSIÓN}

El análisis del PNF mostró que los híbridos de melón tuvieron el mejor PNF fue en el sistema rastrero con 1,95 melones por planta en promedio. Este resultado no fue claro en nuestra investigación. Trabajos similares de investigación, fueron reportados por otros investigadores 
como Monge (2016) quienes mencionaron la existencia de una amplia variabilidad entre los tipos de melón en cuanto a número de frutos por planta $(1,18-3,26)$. Sin embargo, pudieron observar un mayor número de frutos por planta se obtuvo en los tipos de melón amarillo $(3,26)$ y japonés (2.79); mientras que el menor número, con cantaloupe $(1,48)$ y galia $(1,18)$. Estos resultados muestran que en nuestra investigación se obtuvo un mayor número de frutos $(1,95)$ para el tipo cantaloupe. No hemos probado otros tipos de melones.

El análisis de peso de frutos $(\mathrm{kg})$ mostró que el mayor peso correspondió al sistema de tutorado con 1006g en promedio. Monge (2016) y Diaz y Monge (2017) reportaron un peso promedio del fruto de entre 443.29 - 837.03 g y un rendimiento por planta de 685,08-1882,13 g. Así mismos Loor (2015), menciona que los híbridos de melón evaluados en sus investigaciones tuvieron efecto significativo estadísticamente entre las variables: longitud de guías a los 20 y 40 días, número de frutos comerciales y peso promedio del fruto. Es notorio observar que en nuestra investigación se logró obtener un mejor peso de fruta con el sistema tutorado. Cabe mencionar que no hicimos estudios de la firmeza de la pulpa del fruto y porcentaje de sólidos solubles totales. Estudios que podrían realizarse con los mejores cultivares híbridos seleccionados.

Es importante mencionar que si bien no se observaron diferencias significativas en el PNF y PF, fue notorio el buen comportamiento de los cultivares híbridos Kapaz y Karametza respecto del cultivar híbrido Primo, cultivado en la zona. Estos dos cultivares se comportarían mejor en la zona. Asimismo, se observó que son dulces y agradables al paladar, según la opinión de los consumidores que probaron ambos cultivares.

Si bien no se observaron diferencias estadísticas en el volumen de fruto, se pudo apreciar que los cultivares híbridos Kazik, Kazta y Kapaz tuvieron mejor volumen de fruto que Primo y Karametza. Naranjo (2012) en un estudio de cinco híbridos de melón tipo Cantaloupe bajo condiciones controladas en Ecuador, encontró que los cultivares Halona y Maverick fueron los que mostraron los mayores rendimientos, mientras que Wrangler produjo mayor materia vegetal. El cultivar híbrido Halona, además se destacó por su nivel de agrado al consumidor. En nuestro caso se observó nuevamente al cultivar Kapaz con buen volumen de fruto.

Estudios realizados por Enza Zaden (Enza Zaden, 2018) mostraron que el cultivar Kazta se carcatriza por el color anarankado intenso de la pulpa, tiene un fuerte desarrollo vegetativo y crecen bien bajo condiciones de estrés. Tiene alta resistencia al Fusarium oxysporum f.sp. melonis Fom:0.1.2. alta resistencia al Melon Necrotic Spot Virus (MNSV) e inmunidad al Podosphaeria xanthii (ex Sphaerotheca fuliginea) Px:1.2.5. En cambio, Karametza muestra una gran uniformidad de frutos, con alta resistencia al Fusarium oxysporum f.sp. melonis Fom:0.1.2. e inmunidad al Podosphaeria xanthii (ex Sphaerotheca fuliginea) Px:1.2.5. Kazik en cambio tiene un furte desarrollo vegetativo y desarrolla bien en condiciones bajo estrés, sus frutos son medianos y tienen alto contenido de grados Brix y pulpa anaranjada oscura. Alta resistencia al Fusarium oxysporum f.sp. melonis Fom:0.1.2. e inmunidad al Podosphaeria xanthii (ex Sphaerotheca fuliginea) Px:1.2.5.

Se debe resaltar que el cultivo protegido de melón presenta varias ventajas sobre el cultivo a campo abierto, como mayor número de cosechas durante el año, precocidad de la cosecha, economía de agua y de fertilizantes, mayor rendimiento y mejor calidad de los frutos (Vargas et 
al., 2008). En el cultivo bajo ambiente protegido, se alteran las características ambientales de clima y de suelo: hay menor radiación solar global, evapotranspiración y viento, y hay mayor radiación difusa, temperatura y humedad relativa del aire (Martins et al., 1998).En nuestra investigación no evaluamos la firmeza del fruto, pero este carácter es un componente importante en la calidad de los frutos y es una característica intrínseca a la resistencia contra el deterioro físico y mecánico durante su transporte y mercadeo (Gomes et al., 2001). Se considera que la firmeza de la pulpa es una característica ligada a la genética, incluso muchas veces puede ser un indicador del estado de madurez del fruto (Neibauer y Maynard, 2002).

\section{CONCLUSIONES}

Los resultados permitieron concluir que el melón con mejor promedio de número de frutos fue en el sistema rastrero, pero el mejor peso de fruto se obtuvo en el sistema de tutorado.

Los mejores cultivares fueron Kapaz y kazik en ambos sistemas.

La estimación económica mostró que los cultivares en general todas fueron rentables cuando se vendieron a supermercado.

\section{INFORMACIÓN DE FINANCIACIÓN}

Este estudio fue financiado por la Universidad Estatal del Sur de Manabí (UNESUM), Ecuador (PROG-003-PROY-001-DIP-2017).

\section{REFERENCIAS}

Boardman, NE. 2006. Cost-benefit Analysis: Concepts and Practice. 3rd edición. Upper Saddle River, NJ: Prentice Hall, USA. 560 p. Recuperado de https://www.amazon.com/CostBenefit-Analysis-Concepts-Practice/dp/0131435833

Carrillo, R., Carvajal, T., Valarezo, O., Cañarte , E., Mendoza, A., Mendoza, H., . . . Ponce, J. (2010). Buenas Prácticas Agrícolas y Estimación de Costos de Producción para cultivos de ciclo corto en Manabí. Portoviejo, Ecuador. Instituto Nacional Autónomo de Investigaciones Agropecuarias INIAP, Portoviejo. Recuperado de http://repositorio.espam.edu.ec/bitstream/42000/1097/1/TTA15.pdf

Díaz, J., \& Monge, J. (2017). Efecto de la poda y la densidad de siembra sobre el rendimiento y calidad de melón Cantaloupe (Cucumis melo L.) cultivado bajo invernadero. Revista Colombiana de Ciencias Hortícolas 11(1): 1-10. Recuperado de https://revistas.uptc.edu.co/index.php/ciencias_horticolas/article/view/5742

ENZAZADEN. (2018). Forenza. Recuperado de http://www.enzazaden.com.mx/products-andservices/our-products/Forenza

Gabriel, J; Castro, C; Valverde, A; Indacochea, B. 2017. Diseños experimentales: Teoría y práctica para experimentos agropecuarios (en línea). Grupo COMPAS, Universidad Estatal del Sur de Manabí (UNESUM), Jipijapa, Ecuador. 146 p. ISBN 978-9942-750-50-1. $\begin{array}{llllll}\text { Consultado } 11 \text { de } & \text { febrero } & \text { Recuperado }\end{array}$ http://142.93.18.15:8080/jspui/handle/123456789/116 
Gabriel, J; Delvalle, J; Padilla, J; Pincay, Nixon, Ayón, F; Narváez, W; González, A. (2020a). Innovaciones en la matriz productiva hortícola para reducir el efecto del cambio climático en Puerto la Boca, Jipijapa, Ecuador. J. Selva Andina Res. Soc. 11(1): 2-17. http://dx.doi.org/10.36610/j.jsars.2020.110100002.

Gabriel, J.; Pereira, E.; Ayón, F.; Castro, C.; Delvalle-García, I.; Castillo JA. (2020b). Development of an ecological strategy for the control of downy mildew (Pseudoperonospora cubensis) in cucumber cultivation (Cucumis sativus L.). Revista Bionatura 5(2): 1-15. http://dx.doi.org/10.21931/RB/2020.05.02.3

Gomes, J., Menezes, J., Nunes, G., Costa, F. y Souza, P. (2001). Qualidade pós-colheita de melão tipo cantaloupe, colhido em dois es $\neg$ tádios de maturação. Horticultura Brasileira 19(3): 356-360.

Gómez, J. (2017). Área melonera no crece en Ecuador por falta de incentivos. Recuperado de http://actoresproductivos.com/2017/11/20/area-melonera-no-crece-en-ecuador/

Loor, H. (2015). Comportamiento agronómico de tres hibridos de melón (Cucumis melo L.) bajo dos densidades poblacionales. Guayaquil - Ecuador: Universidad de Guayaquil. Facultad de Ciencias Agrarias. Recuperado de http://repositorio.ug.edu.ec/handle/redug/7495

Martel Moreno, J. 1999. Fórmulas generales para determinación de áreas y volúmenes. El Guiniguada 8/9: 291-317. $\quad$ Recuperado de https://www.yumpu.com/es/document/read/14499221/formulas-generales-para-ladeterminacion-de-areas-y-volumenes

Martins, S. R., Peil, R. M., Schwengber, J. E., As $\neg$ sis, F. N. y Méndez, M. E. (1998). Produção de melão em função de diferentes sistemas de condução de plantas em ambiente pro $\neg$ tegido. Horticultura Brasileira 16(1): 24-30.

Monge, J. (2016). Evaluación de 70 genotipos de melón (Cucumis melo l.) cultivados bajo invernadero en costa rica. InterSedes 17(36): 2215-2458. http://dx.doi.org/10.15517/isucr.v17i36.26944

Naranjo, A. (2012). "Evaluación agronómica y de calidad en diferentes híbridos de melón Cucumis melo grupo Cantaloupe bajo condiciones controladas en el valle de Tumbaco". Quito - Ecuador : Universidad San Francisco de Quito. Recuperado de http://repositorio.usfq.edu.ec/handle/23000/1483

Neibauer, J. y Maynard, E. (2002). Información poscosecha: normas de calidad del USDA. Disponible en http://www.hort.purdue.edu/prod_uality/commodities/muskmelon.html

Orrala, N., Borbor, E., \& Dominguez, G. (2013). Empleo de tecnologías limpias para el manejo de problemas fitosanitarios en el cultivo de melón Cucumis melo l. comuna Río Verde, Santa Elena. UPSE: 1-10. Recuperado de https://repositorio.upse.edu.ec/handle/46000/910

Ramírez, G. (2014). Análisis económico de la producción de sandía (Citrullus lanatus) injertada sobre patrones de calabaza en la provincia de Santa Elena. UPSE: 1-10. Recuperado de https://repositorio.upse.edu.ec/xmlui/handle/46000/2250 
UNESUM-CIENCIAS: REVISTA CIENTÍFICA MULTIDISCIPLINARIA

Nuevos cultivares de melón (Cucumis melo L.) para invernadero en Puerto La Boca, Manabí

SAS University (2019). An Introduction to SAS University Edition (en línea). Consultado 03 febrero 2020. Recuperado de https://www.oreilly.com/library/view/an-introductionto/9781629600079/

Vargas, P. F., Castoldi, R., Charlo, H. C. y Braz, L. T. (2008). Qualidade de melão ren $\neg$ dilhado (Cucumis melo L.) em função do sistema de cultivo. Ciência e Agrotecnologia 32(1), 137142. https://doi.org/10.1590/S1413-70542008000100020. 